\title{
Design and evaluation of synthetic terminators for regulating mammalian cell transgene expression
}

Joseph K. Cheng',3, Nicholas J. Morse ${ }^{1}$, James M. Wagner ${ }^{1}$, Scott K. Tucker ${ }^{2}$, and Hal S. Alper $^{1,2, *}$

${ }^{1}$ McKetta Department of Chemical Engineering, The University of Texas at Austin, 200 E Dean Keeton St. Stop C0400 Austin, Texas 78712

${ }^{2}$ Institute for Cellular and Molecular Biology, The University of Texas at Austin, 2500 Speedway Avenue, Austin, Texas 78712

${ }^{3}$ Current Address: Ben Towne Center for Childhood Cancer Research, Seattle Children's Research Institute, 1100 Olive Way, Suite 100, Seattle, Washington 98101

*Correspondence and requests for materials should be addressed to H.S.A. (halper@ che.utexas.edu)

\section{Supporting Information}




\section{Accession codes of nucleotide sequences used in this manuscript}

Human cytomegalovirus: M60321.1

Simian Virus 40: J02400.1

Human chromosomes, GRCh38.p2 assembly: NC_000006.12 (EEF1A1), NC_000007.14 (ACTB), NC_000012.12 (GAPDH and RPL41), and NC_000017.11 (EIF4AI) 


\section{Equations}

Because actinomycin D affects transcription of all RNA, any reference gene we chose would have had its own intrinsic degradation rate and thus fail to normalize the data. Instead, we calculated relative degradation by measuring the difference in degradation constants between our samples and the positive SV40 terminator control.

$$
\begin{gathered}
R N A_{1, t}=R N A_{1, t=0} e^{-\lambda_{1} t} \\
R N A_{2, t}=R N A_{2, t=0} e^{-\lambda_{2} t} \\
\frac{R N A_{2, t}}{R N A_{1, t}}=\frac{R N A_{2, t=0}}{R N A_{1, t=0}} e^{-\left(\lambda_{2}-\lambda_{1}\right) t} \\
\ln \frac{R N A_{2, t}}{R N A_{1, t}}=-\left(\lambda_{2}-\lambda_{1}\right) t+\ln \frac{R N A_{2, t=0}}{R N A_{1, t=0}}
\end{gathered}
$$

This difference has an inverse linear relationship with steady state fluorescence levels, allowing us to determine whether the differences in GFP arise from differences in degradation rate.

$$
\begin{gathered}
\frac{d[m R N A]}{d t}=r_{\text {promoter }}-\lambda[m R N A] \\
\frac{d[G F P]}{d t}=k_{\text {translation }}[m R N A]-\delta_{G F P}[G F P] \\
{[m R N A]_{\text {S.S. }}=\frac{r_{\text {promoter }}}{\lambda}=\frac{\delta_{G F P}}{k_{\text {translation }}}[G F P]_{S . S}} \\
\lambda_{2}-\lambda_{1}=r_{\text {promoter }} * \frac{k_{\text {translation }}}{\delta_{\text {GFP }}}\left[\frac{1}{[G F P]_{2}}-\frac{1}{[G F P]_{1}}\right]
\end{gathered}
$$

Therefore, by assuming promoter expression (same promoter), GFP translation (same Kozak sequence), and GFP degradation (same peptide sequence) remain constant, we can determine if terminator sequences affect GFP by affecting degradation rate. 


\section{Supporting Tables and Graphics}

Table S1: Terminators compared with the SV40 terminator based on hrGFP expression driven by EIF4A1-derived promoter in HT1080. Statistical significance determined by ANOVA and Tukey's HSD post-hoc testing. Expression difference corresponds to the difference of log mean hrGFP expression of the particular terminator relative to the SV40 terminator.

\begin{tabular}{|l|r|l|}
\hline \multicolumn{1}{|l|}{ terminator pair } & \multicolumn{1}{l|}{ expression difference } & \multicolumn{1}{l|}{ padjusted } \\
\hline HT1080.WT-f.SV40pA & -1.781 & 0.000 \\
\hline m.SV40pA.1-f.SV40pA & -0.312 & 0.000 \\
\hline m.SV40pA.2-f.SV40pA & -0.097 & 0.964 \\
\hline m.SV40pA.3-f.SV40pA & -0.078 & 0.999 \\
\hline m.SV40pA.4-f.SV40pA & -0.028 & 1.000 \\
\hline no.terminator-f.SV40pA & -1.187 & 0.000 \\
\hline T.ACTB.1-f.SV40pA & -0.363 & 0.000 \\
\hline T.ACTB.2-f.SV40pA & -0.812 & 0.000 \\
\hline T.ACTB.3-f.SV40pA & 0.013 & 1.000 \\
\hline T.ACTB.4-f.SV40pA & 0.042 & 1.000 \\
\hline T.ACTB.f-f.SV40pA & -0.387 & 0.000 \\
\hline T.EEF1A1.1-f.SV40pA & -0.411 & 0.000 \\
\hline T.EEF1A1.2-f.SV40pA & -0.252 & 0.000 \\
\hline T.EEF1A1.3-f.SV40pA & -0.537 & 0.000 \\
\hline T.EEF1A1.4-f.SV40pA & -0.432 & 0.000 \\
\hline T.GAPDH.1-f.SV40pA & -0.432 & 0.000 \\
\hline T.GAPDH.2-f.SV40pA & 0.038 & 1.000 \\
\hline Tm.synth.01-f.SV40pA & -0.128 & 0.520 \\
\hline Tm.synth.02-f.SV40pA & -0.075 & 1.000 \\
\hline Tm.synth.03-f.SV40pA & -0.088 & 0.992 \\
\hline Tm.synth.04-f.SV40pA & -0.146 & 0.216 \\
\hline Tm.synth.05-f.SV40pA & -0.180 & 0.016 \\
\hline Tm.synth.06-f.SV40pA & -0.107 & 0.880 \\
\hline Tm.synth.07-f.SV40pA & -0.081 & 0.998 \\
\hline Tm.synth.08-f.SV40pA & -0.650 & 0.000 \\
\hline Tm.synth.09-f.SV40pA & -0.091 & 0.987 \\
\hline Tm.synth.10-f.SV40pA & -0.115 & 0.757 \\
\hline Tm.synth.11-f.SV40pA & -0.732 & 0.000 \\
\hline Tm.synth.12-f.SV40pA & -0.190 & 0.007 \\
\hline Tm.synth.13-f.SV40pA & -0.018 & 1.000 \\
\hline Tm.synth.14-f.SV40pA & -0.145 & 0.225 \\
\hline Tm.synth.15-f.SV40pA & -0.214 & 0.001 \\
\hline Tm.synth.16-f.SV40pA & -0.442 & 0.000 \\
\hline Tm.synth.17-f.SV40pA & -0.437 & 0.000 \\
\hline Tm.synth.18-f.SV40pA & -0.642 & 0.000 \\
\hline Tm.synth.19-f.SV40pA & -0.559 & 0.000 \\
\hline Tm.synth.20-f.SV40pA & -0.107 & 0.876 \\
\hline Tm.synth.21-f.SV40pA & -0.566 & 0.000 \\
\hline Tm.synth.22-f.SV40pA & -0.802 & 0.000 \\
\hline Tm.synth.23-f.SV40pA & -0.715 & 0.000 \\
\hline Tm.synth.24-f.SV40pA & -0.574 & 0.000 \\
\hline Tm.synth.25-f.SV40pA & -0.145 & 0.229 \\
\hline Tm.synth.26-f.SV40pA & -0.596 & 0.000 \\
\hline Tm.synth.27-f.SV40pA & -0.099 & 0.952 \\
\hline Tm.synth.28-f.SV40pA & -0.046 & 1.000 \\
\hline
\end{tabular}




\begin{tabular}{|l|r|l|}
\hline terminator pair & expression difference & padjusted \\
\hline Tm.synth.29-f.SV40pA & -0.494 & 0.000 \\
\hline Tm.synth.30-f.SV40pA & -0.444 & 0.000 \\
\hline
\end{tabular}

Table S2: Terminators compared with the SV40 terminator based on hrGFP expression driven by EIF4A1-derived promoter with sub-population gating and an RFP transfection marker (constitutive mStrawberry expression driven by CMV promoter) in HT1080. Expression difference corresponds to the difference of log mean hrGFP expression of the particular terminator relative to the SV40 terminator.

\begin{tabular}{|l|r|}
\hline \multicolumn{1}{|l|}{ terminator pair } & expression difference \\
\hline no.terminator-f.SV40pA & -1.856 \\
\hline T.GAPDH.1-f.SV40pA & -0.312 \\
\hline T.GAPDH.2-f.SV40pA & 0.059 \\
\hline Tm.synth.02-f.SV40pA & -0.172 \\
\hline Tm.synth.15-f.SV40pA & -0.188 \\
\hline Tm.synth.17-f.SV40pA & -0.399 \\
\hline Tm.synth.22-f.SV40pA & -1.017 \\
\hline
\end{tabular}

Table S3: Terminators compared with the SV40 terminator based on SEAP expression in HT1080. Statistical significance determined by ANOVA and Tukey's HSD posthoc testing. Expression difference corresponds to the difference in SEAP productivity of the particular terminator relative to SV40.

\begin{tabular}{|l|r|l|}
\hline \multicolumn{2}{|c|}{ expression difference } & padjusted \\
\hline no.terminator-f.SV40pA & -0.120 & 0.000 \\
\hline T.GAPDH.2-f.SV40pA & -0.057 & 0.000 \\
\hline T.ACTB.4-f.SV40pA & -0.020 & 0.011 \\
\hline Tm.synth.08-f.SV40pA & -0.112 & 0.000 \\
\hline Tm.synth.09-f.SV40pA & -0.079 & 0.000 \\
\hline Tm.synth.10-f.SV40pA & -0.109 & 0.000 \\
\hline Tm.synth.18-f.SV40pA & -0.100 & 0.000 \\
\hline Tm.synth.19-f.SV40pA & -0.014 & 0.384 \\
\hline Tm.synth.20-f.SV40pA & 0.009 & 0.914 \\
\hline Tm.synth.23-f.SV40pA & -0.111 & 0.000 \\
\hline Tm.synth.24-f.SV40pA & -0.064 & 0.000 \\
\hline Tm.synth.25-f.SV40pA & -0.041 & 0.000 \\
\hline Tm.synth.26-f.SV40pA & -0.099 & 0.000 \\
\hline Tm.synth.27-f.SV40pA & -0.052 & 0.000 \\
\hline Tm.synth.28-f.SV40pA & -0.048 & 0.000 \\
\hline
\end{tabular}

Table S4: Terminators compared with the SV40 terminator based on hrGFP expression driven by a strong promoter (pEEF1A1) in HT1080. Statistical significance determined by ANOVA and Tukey's HSD post-hoc testing. Expression difference corresponds to the difference of log mean hrGFP expression of the particular terminator relative to SV40.

\begin{tabular}{|l|c|l|}
\hline terminator pair & expression difference & \multicolumn{1}{c|}{ padjusted } \\
\hline HT1080.WT-pEEF1A1.f.SV40pA & -2.443 & 0.000 \\
\hline
\end{tabular}




\begin{tabular}{|l|r|l|}
\hline pEEF1A1.no.terminator-pEEF1A1.f.SV40pA & -1.017 & 0.000 \\
\hline pEEF1A1.T.GAPDH.2-pEEF1A1.f.SV40pA & -0.395 & 0.000 \\
\hline pEEF1A1.T.ACTB.4-pEEF1A1.f.SV40pA & -0.255 & 0.004 \\
\hline pEEF1A1.Tm.synth.08-pEEF1A1.f.SV40pA & -0.197 & 0.084 \\
\hline pEEF1A1.Tm.synth.09-pEEF1A1.f.SV40pA & -0.066 & 0.999 \\
\hline pEEF1A1.Tm.synth.10-pEEF1A1.f.SV40pA & 0.106 & 0.907 \\
\hline pEEF1A1.Tm.synth.18-pEEF1A1.f.SV40pA & -0.228 & 0.019 \\
\hline pEEF1A1.Tm.synth.19-pEEF1A1.f.SV40pA & 0.022 & 1.000 \\
\hline pEEF1A1.Tm.synth.20-pEEF1A1.f.SV40pA & 0.107 & 0.901 \\
\hline pEEF1A1.Tm.synth.23-pEEF1A1.f.SV40pA & -0.259 & 0.004 \\
\hline pEEF1A1.Tm.synth.24-pEEF1A1.f.SV40pA & 0.070 & 0.998 \\
\hline pEEF1A1.Tm.synth.25-pEEF1A1.f.SV40pA & -0.042 & 1.000 \\
\hline pEEF1A1.Tm.synth.26-pEEF1A1.f.SV40pA & -0.312 & 0.000 \\
\hline pEEF1A1.Tm.synth.27-pEEF1A1.f.SV40pA & 0.150 & 0.439 \\
\hline pEEF1A1.Tm.synth.28-pEEF1A1.f.SV40pA & 0.063 & 0.999 \\
\hline
\end{tabular}

Table S5: Terminators compared with the SV40 terminator based on hrGFP expression driven by EIF4A1-derived promoter in HEK293. Statistical significance determined by ANOVA and Tukey's HSD post-hoc testing. Expression difference corresponds to the difference of log mean hrGFP expression of the particular terminator relative to SV40.

\begin{tabular}{|c|c|c|}
\hline terminator pair & expression difference & padjusted \\
\hline HEK293-f.SV40pA & -0.449 & 0.000 \\
\hline no.terminator-f.SV40pA & -0.301 & 0.000 \\
\hline T.GAPDH.2-f.SV40pA & 0.073 & 0.183 \\
\hline T.ACTB.4-f.SV40pA & 0.008 & 1.000 \\
\hline Tm.synth.08-f.SV40pA & -0.157 & 0.000 \\
\hline Tm.synth.09-f.SV40pA & -0.043 & 0.915 \\
\hline Tm.synth.10-f.SV40pA & -0.039 & 0.957 \\
\hline Tm.synth.18-f.SV40pA & -0.084 & 0.060 \\
\hline Tm.synth.19-f.SV40pA & 0.046 & 0.857 \\
\hline Tm.synth.20-f.SV40pA & 0.049 & 0.804 \\
\hline Tm.synth.23-f.SV40pA & -0.145 & 0.000 \\
\hline Tm.synth.24-f.SV40pA & 0.031 & 0.996 \\
\hline Tm.synth.25-f.SV40pA & -0.086 & 0.049 \\
\hline Tm.synth.26-f.SV40pA & -0.116 & 0.001 \\
\hline Tm.synth.27-f.SV40pA & 0.015 & 1.000 \\
\hline Tm.synth.28-f.SV40pA & -0.006 & 1.000 \\
\hline
\end{tabular}

Table S6: Differential expression between a particular DSE and the DSE consensus from ANOVA analysis with Tukey's HSD post-hoc testing based on hrGFP expression driven by the pEIF4A1.636 promoter.

\begin{tabular}{|l|c|l|}
\hline pairwise comparison & differential expression & padjusted \\
\hline Levitt-consensus & 0.140 & 0.035 \\
\hline MC4R-consensus & 0.220 & 0.002 \\
\hline CstF64.RRM-consensus & 0.271 & 0.000 \\
\hline consensus+Levitt-consensus & 0.570 & 0.000 \\
\hline
\end{tabular}


Table S7: ANOVA analysis with Tukey's HSD post-hoc testing of the spacer 2, polyadenylation site, and DSE impact on hrGFP expression driven by the pEIF4A1.636 promoter.

\begin{tabular}{|l|l|}
\hline Factor/interaction & p-value \\
\hline spacer.2 & 0.033 \\
\hline PA.site & 0.919 \\
\hline DSE & $<2.20 \mathrm{E}-16$ \\
\hline spacer.2:PA.site & $9.96 \mathrm{E}-11$ \\
\hline spacer.2:DSE & 0.349 \\
\hline PA.site:DSE & 0.779 \\
\hline spacer.2:PA.site:DSE & $2.52 \mathrm{E}-10$ \\
\hline
\end{tabular}

Table S8: ANOVA analysis with Tukey's HSD post-hoc testing of the spacer 2, polyadenylation site, and DSE impact on SEAP expression driven by the pEIF4A1.636 promoter.

\begin{tabular}{|l|l|}
\hline Factor/interaction & p-value \\
\hline spacer.2 & 0.000 \\
\hline PA.site & 0.755 \\
\hline DSE & $3.351 \mathrm{E}-08$ \\
\hline spacer.2:PA.site & $4.94 \mathrm{E}-05$ \\
\hline spacer.2:DSE & 0.022 \\
\hline PA.site:DSE & 0.503 \\
\hline spacer.2:PA.site:DSE & $1.23 \mathrm{E}-03$ \\
\hline
\end{tabular}

Table S9: ANOVA analysis with Tukey's HSD post-hoc testing of the spacer 2, polyadenylation site, and DSE impact on hrGFP expression driven by the pEEF1A1.1356 promoter.

\begin{tabular}{|l|l|}
\hline Factor/interaction & p-value \\
\hline spacer.2 & 0.116 \\
\hline PA.site & 0.539 \\
\hline DSE & $1.08 \mathrm{E}-12$ \\
\hline spacer.2:PA.site & 0.527 \\
\hline spacer.2:DSE & 0.035 \\
\hline PA.site:DSE & $9.01 \mathrm{E}-05$ \\
\hline spacer.2:PA.site:DSE & 0.361 \\
\hline
\end{tabular}

Table S10: Fully endogenous/synthetic genetic elements compared with viral-derived elements based on hrGFP expression in HT1080. rpCMV corresponds to reference CMV promoter, pEEF1A1 corresponds to the 1356-bp promoter derived from EEF1A1. Statistical significance determined by ANOVA and Tukey's HSD post-hoc testing. Expression difference corresponds to the difference of log mean hrGFP expression of the particular terminator relative to f.SV40pA.

\begin{tabular}{|l|r|l|}
\hline terminator pair & expression difference & padjusted \\
\hline rpCMV.f.SV40pA-HT1080.WT & 1.975 & 0.000 \\
\hline
\end{tabular}




\begin{tabular}{|l|r|l|}
\hline rpCMV.f.SV40pA-pEEF1A1.no.terminator & 0.545 & 0.000 \\
\hline rpCMV.f.SV40pA-pEEF1A1.f.SV40pA & -0.471 & 0.000 \\
\hline rpCMV.f.SV40pA-pEEF1A1.T.GAPDH.2 & -0.076 & 0.915 \\
\hline rpCMV.f.SV40pA-pEEF1A1.T.ACTB.4 & -0.216 & 0.041 \\
\hline rpCMV.f.SV40pA-pEEF1A1.Tm.synth.13 & -0.343 & 0.000 \\
\hline
\end{tabular}


Table S11: Table of native/endogenous terminator sequences with putative spacer regions in lower case.

\begin{tabular}{|c|c|c|}
\hline terminator & sequence $5^{\prime}>3$ & length \\
\hline f.SV40pA & $\begin{array}{l}\text { CAGACATGATAAGATACATTGATGAGTTTGGACAAACCACAACTAGAATGCAGTGAAAAAAAT } \\
\text { GCTTTATTTGTGAAATTTGTGATGCTATTGCTTTATTTGTAAccattataagctgcAATAAAcaagttaacaacA } \\
\text { AcaattgcattcattTTATGTTCAGGTTCAGGGGGAGGTGTGGGAGGTTTTTAAAGCAAGTAAAACCT } \\
\text { CTACAAATGTGGTA }\end{array}$ & 222 \\
\hline m.SV40pA.1 & TGTAAccattataagctgcAATAAAcaagttaacaacAAcaattgcattcatttTATGTTTC & 62 \\
\hline m.SV40pA.2 & $\begin{array}{l}\text { TGTAAccattataagctgcAATAAAcaagttaacaacAAcaattgcattcatttTATGTTTCAGGTTCAGGGGGAGGTGT } \\
\text { GGGAGGTTTTTT }\end{array}$ & 92 \\
\hline m.SV40pA.3 & $\begin{array}{l}\text { CAGACATGATAAGATACATTGATGAGTTTGGACAAACCACAACTAGAATGCAGTGAAAAAAAT } \\
\text { GCTTTATTTGTGAAATTTGTGATGCTATTGCTTTATTTGTAAccattataagctgcAATAAAcaagttaacaacA } \\
\text { AcaattgcattcatttTATGTTTCAGGTTCAGGGGGAGGTGTGGGAGGTTTTTT }\end{array}$ & 192 \\
\hline m.SV40pA.4 & $\begin{array}{l}\text { TGTGATGCTATTGCTTTATTTGTAAccattataagctgcAATAAAcaagttaacaacAAcaattgcattcatttTATGTTTC } \\
\text { AGGTTCAGGGGGAGGTGTGGGAGGTTTTTTAAAGCAAGTAAAACCTCTACAAATGTGGTA }\end{array}$ & 142 \\
\hline T.EEF1A1.1 & TGGTATtcattacaaacttgctcactacAATAAAtgaattttaagctttAAgatgaagtggcaTTTCTTTT & 71 \\
\hline T.EEF1A1.2 & $\begin{array}{l}\text { TGGTATtcattacaaacttgctcactacAATAAAtgaattttaagctttAAgatgaagtggcaTTTCTTTTAACAGTTACTATGT } \\
\text { TGGAATTGGTTACAAATTTTGGAGTGGATTTCAAAAGTGAGAGCTAACTTCAGTTGATTTCAAG } \\
\text { GTAGTGCTTGGCTTTTTTTGTTTA }\end{array}$ & 173 \\
\hline T.EEF1A1.3 & $\begin{array}{l}\text { TGTGAAACCCAGTGTCTTAGACAACTGTGGCTTGAGCACCACCTGCTGGTATtcattacaaacttgctcacta } \\
\text { cAATAAAtgaattttaagctttAAgatgaagtggcaTTTCTTTT }\end{array}$ & 117 \\
\hline T.EEF1A1.4 & $\begin{array}{l}\text { TGTGAAACCCAGTGTCTTAGACAACTGTGGCTTGAGCACCACCTGCTGGTATtcattacaaacttgctcacta } \\
\text { cAATAAAtgaatttaagctttAAgatgaagtggcaTTTCTTTTAACAGTTACTATGTTGGAATTGGTTACAAATT } \\
\text { TTGGAGTGGATTTCAAAAGTGAGAGCTAACTTCAGTTGATTTCAAGGTAGTGCTTGGCTTTTTT } \\
\text { TGTTTA }\end{array}$ & 219 \\
\hline T.GAPDH.1 & $\begin{array}{l}\text { TGTCATGTACcatcAATAAAgtaccetgtgctcaaccagtTActtgtcctgtcttattctagGGTCTGGGGCAGAGGGGAGG } \\
\text { GAAGCTGGGCTTGTGTC }\end{array}$ & 99 \\
\hline T.GAPDH.2 & $\begin{array}{l}\text { TGTAGACCCCTTGAAGAGGGGAGGGGCCTAGGGAGCCGCACCTTGTCATGTACcatcAATAAAgta } \\
\text { cectgtgctcaaccagtTActtgtcetgtcttattctagGGTCTGGGGCAGAGGGGAGGGAAGCTGGGCTTGTGTC }\end{array}$ & 142 \\
\hline T.ACTB.f & 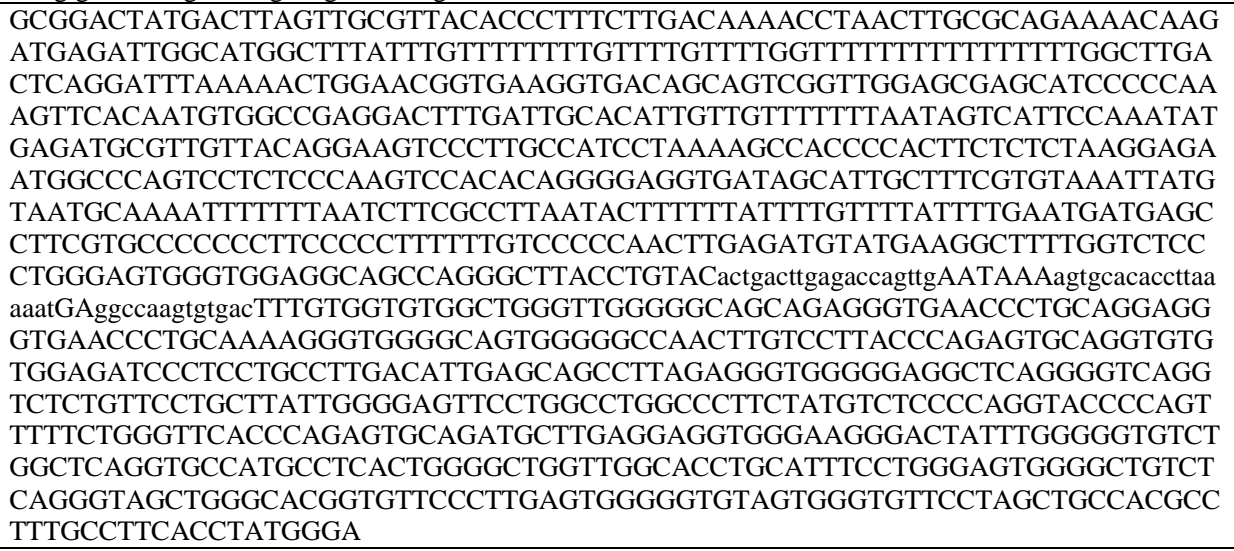 & 1065 \\
\hline Т.АСТВ. 1 & $\begin{array}{l}\text { GCGGACTATGACTTAGTTGCGTTACACCCTTTCTTGACAAAACCTAACTTGCGCAGAAAACAAG } \\
\text { ATGAGATTGGCATGGCTTTATTTGTTTTTTTTTTTGTTTGGTTTTTTTTTTTTTTGGCTTGA } \\
\text { CTCAGGATTAAAAACTGGAACGGTGAAGGTGACAGCAGTCGGTTGGAGCGAGCATCCCCCAA } \\
\text { AGTTCACAATGTGGCCGAGGACTTTGATTGCACATTGTTGTTTTTTTAATAGTCATTCCAAATAT } \\
\text { GAGATGCGTTGTTACAGGAAGTCCCTTGCCATCCTAAAAGCCACCCCACTTCTCTCTAAGGAGA } \\
\text { ATGGCCCAGTCTCTCCCAAGTCCACACAGGGGAGGTGATAGCATTGCTTTCGTGTAAATTATG } \\
\text { TAATGCAAAATTTTTTAATCTTCGCCTTAATACTTTTTTATTTTGTTTTATTTTGAATGATGAGC } \\
\text { CTTCGTGCCCCCCCTTCCCCCTTTTTTGTCCCCCAACTTGAGATGTATGAAGGCTTTTGGTCTCC } \\
\text { CTGGGAGTGGGTGGAGGCAGCCAGGGCTTACCTGTACactgacttgagaccagttgAATAAAagtgcacaccttaa } \\
\text { aaatGAggcaagtgtgacTTTGTGGTGTGGCTGGGTTGGGGGCAGCAGAGGGTGAACCCTGCAGGAGG } \\
\text { GTGAACCCTGCAAAAGGGTGGGGCAGTGGGGGCCAAC }\end{array}$ & 700 \\
\hline
\end{tabular}




\begin{tabular}{|c|c|c|}
\hline terminator & sequence $5^{\prime}>3^{\prime}$ & length \\
\hline Т.АСТВ. 2 & $\begin{array}{l}\text { GCGGACTATGACTTAGTTGCGTTACACCCTTTCTTGACAAAACCTAACTTGCGCAGAAAACAAG } \\
\text { ATGAGATTGGCATGGCTTTATTTGTTTTTTTTGTTTTGTTTTGGTTTTTTTTTTTTTTTTGGCTTGA } \\
\text { CTCAGGATTAAAAACTGGAACGGTGAAGGTGACAGCAGTCGGTTGGAGCGAGCATCCCCCAA } \\
\text { AGTTCACAATGTGGCCGAGGACTTTGATTGCACATTGTTGTTTTTTAATAGTCATTCCAAATAT } \\
\text { GAGATGCGTTGTTACAGGAAGTCCCTTGCCATCCTAAAAGCCACCCCACTTCTCTCTAAGGAGA } \\
\text { ATGGCCCAGTCCTCTCCCAAGTCCACACAGGGGAGGTGATAGCATTGCTTTCGTGTAAATTATG } \\
\text { TAATGCAAAATTTTTTAATCTTCGCCTTAATACTTTTTTATTTGTTTATTTGAATGATGAGC } \\
\text { CTTCGTGCCCCCCCTTCCCCCTTTTTTGTCCCCCAACTTGAGATGTATGAAGGCTTTTGGTCTCC } \\
\text { CTGGGAGTGGGTGGAGGCAGCCAGGGCTTACCTGTACactgacttgagaccagttgAATAAAagtgcacacettaa } \\
\text { aaatGAggcaagtgtgacTTTGTGGTGTGGCTGGGTTGGGGG }\end{array}$ & 637 \\
\hline Т.АСТВ. 3 & $\begin{array}{l}\text { TTGCTTTCGTGTAAATTATGTAATGCAAAATTTTTTTAATCTTCGCCTTAATACTTTTTTATTTTG } \\
\text { TTTTATTTTGAATGATGAGCCTTCGTGCCCCCCCTTCCCCCTTTTTGTCCCCCAACTTGAGATGT } \\
\text { ATGAAGGCTTTTGGTCTCCCTGGGAGTGGGTGGAGGCAGCCAGGGCTTACCTGTACactgacttgaga } \\
\text { ccagttgAATAAAagtgcacaccttaaaaatGAggccaagtgtgacTTTGTGGTGTGGCTGGGTTGGGGGCAGCAGA } \\
\text { GGGTGAACCCTGCAGGAGGGTGAACCCTGCAAAAGGGTGGGGCAGTGGGGGCCAAC }\end{array}$ & 333 \\
\hline T.АСТВ.4 & $\begin{array}{l}\text { TTGCTTTCGTGTAAATTATGTAATGCAAAATTTTTTTAATCTTCGCCTTAATACTTTTTTATTTTG } \\
\text { TTTTATTTTGAATGATGAGCCTTCGTGCCCCCCCTTCCCCCTTTTTGTCCCCCAACTTGAGATGT } \\
\text { ATGAAGGCTTTTGGTCTCCCTGGGAGTGGGTGGAGGCAGCCAGGGCTTACCTGTACactgacttgaga } \\
\text { ccagttgAATAAAagtgcacaccttaaaaatGAggccaagtgtgacTTTGTGGTGTGGCTGGGTTGGGGG }\end{array}$ & 270 \\
\hline
\end{tabular}

Table S12: Table of synthetic terminator sequences.

\begin{tabular}{|c|c|c|}
\hline terminator & sequence $5^{\prime}>3$ & length \\
\hline Tm.synth.1 & $\begin{array}{l}\text { TGTAGACCCCTTGAAGAGGGGAGGGGCCTAGGGAGCCGCACCTTGTCATGTACcatcAATAAAgta } \\
\text { ccetgtgctcaaccagtTActtgtcctgtcttattctagTGTGTTTT }\end{array}$ & 113 \\
\hline Tm.synth. 2 & $\begin{array}{l}\text { TGTAGACCCCTTGAAGAGGGGAGGGGCCTAGGGAGCCGCACCTTGTCATGTACcatcAATAAAgta } \\
\text { ccctgtgctcaaccagtTActtgtcctgtcttattctagTCTGTGTGTTGGTTTTTTGTGTG }\end{array}$ & 128 \\
\hline Tm.synth.3 & $\begin{array}{l}\text { TGTAGACCCCTTGAAGAGGGGAGGGGCCTAGGGAGCCGCACCTTGTCATGTACcatcAATAAAgta } \\
\text { cectgtgctcaaccagtTActtgtcctgtcttattctagTGTGTTTTTCTGTGTGTTGGTTTTTTGTGTG }\end{array}$ & 136 \\
\hline Tm.synth.4 & $\begin{array}{l}\text { TGTAATGTAATGTAATGTAAcatcAATAAAgtaccetgtgctcaaccagtTActtgtcctgtcttattctagGGTCTGGGGC } \\
\text { AGAGGGGAGGGAAGCTGGGCTTGTGTC }\end{array}$ & 109 \\
\hline Tm.synth.5 & TGTAATGTAATGTAATGTAAcatcAATAAAgtaccctgtgctcaaccagtTActtgtcctgtcttattctagTGTGTTTT & 80 \\
\hline Tm.synth.6 & $\begin{array}{l}\text { TGTAATGTAATGTAATGTAAcatcAATAAAgtaccetgtgctcaaccagtTActtgtcctgtcttattctagTCTGTGTGTTG } \\
\text { GTTTTTTGTGTG }\end{array}$ & 95 \\
\hline Tm.synth.7 & $\begin{array}{l}\text { TGTAATGTAATGTAATGTAAcatcAATAAAgtaccetgtgctcaaccagtTActtgtcctgtcttattctagTGTGTTTTTCT } \\
\text { GTGTGTTGGTTTTTTGTGTG }\end{array}$ & 103 \\
\hline Tm.synth.8 & TGTAATGTAATGTAATGTAAAATAAAgtaccetgtgctcaaccagtTATGTGTTTT & 56 \\
\hline Tm.synth.9 & TGTAATGTAATGTAATGTAAAATAAAgtaccctgtgctcaaccagtTATCTGTGTGTTGGTTTTTTGTGTG & 71 \\
\hline Tm.synth.10 & $\begin{array}{l}\text { TGTAATGTAATGTAATGTAAAATAAAgtaccetgtgctcaaccagtTATGTGTTTTTCTGTGTGTTGGTTTTT } \\
\text { TGTGTG }\end{array}$ & 79 \\
\hline Tm.synth.11 & TGGTATtcattacaaacttgctcactacAATAAAtgaattttaagctttAAgatgaagtggcaTGTGTTTT & 71 \\
\hline Tm.synth.12 & $\begin{array}{l}\text { TGGTATtcattacaaacttgctcactacAATAAAtgaattttaagctttAAgatgaagtggcaTCTGTGTGTTGGTTTTTTGTGT } \\
\text { G }\end{array}$ & 86 \\
\hline Tm.synth.13 & $\begin{array}{l}\text { TGGTATtcattacaaacttgctcactacAATAAAtgaattttaagctttAAgatgaagtggcaTGTGTTTTTCTGTGTGTTGGTT } \\
\text { TTTTGTGTG }\end{array}$ & 94 \\
\hline Tm.synth.14 & $\begin{array}{l}\text { TGTAATGTAATGTAATGTAAtcattacaaacttgctcactacAATAAAtgaattttaagctttAAgatgaagtggcaTTTCTTTT } \\
\text { AACAGTTACTATGTTGGAATTGGTTACAAATTTTGGAGTGGATTTCAAAAGTGAGAGCTAACTT } \\
\text { CAGTTGATTTCAAGGTAGTGCTTGGCTTTTTTGTTTA }\end{array}$ & 187 \\
\hline Tm.synth.15 & $\begin{array}{l}\text { TGTAATGTAATGTAATGTAAtcattacaaacttgctcactacAATAAAtgaattttaagctttAAgatgaagtggcaTGTGTTT } \\
\text { T }\end{array}$ & 85 \\
\hline Tm.synth.16 & $\begin{array}{l}\text { TGTAATGTAATGTAATGTAAtcattacaaacttgctcactacAATAAAtgaattttaagctttAAgatgaagtggcaTCTGTGT } \\
\text { GTTGGTTTTTTGTGTG }\end{array}$ & 100 \\
\hline Tm.synth.17 & $\begin{array}{l}\text { TGTAATGTAATGTAATGTAAtcattacaaacttgctcactacAATAAAtgaattttaagctttAAgatgaagtggcaTGTGTTT } \\
\text { TTCTGTGTGTTGGTTTTTTGTGTG }\end{array}$ & 108 \\
\hline Tm.synth.18 & TGTAATGTAATGTAATGTAAAATAAAtgaattttaagctttAATGTGTTTT & 51 \\
\hline Tm.synth.19 & TGTAATGTAATGTAATGTAAAATAAAtgaattttaagctttAATCTGTGTGTTGGTTTTTTGTGTG & 66 \\
\hline Tm.synth.20 & $\begin{array}{l}\text { TGTAATGTAATGTAATGTAAAATAAAtgaattttaagctttAATGTGTTTTTCTGTGTGTTGGTTTTTTGTG } \\
\text { TG }\end{array}$ & 74 \\
\hline Tm.synth.21 & $\begin{array}{l}\text { TGTAATGTAATGTAATGTAAAATAAAagtgcacaccttaaaaatGATGTGTTTTTCTGTGTGTTGGTTTTTT } \\
\text { GTGTG }\end{array}$ & 77 \\
\hline Tm.synth.22 & $\begin{array}{l}\text { TGTAATGTAATGTAATGTAAAATAAAagtgcacaccttaaaaatCATGTGTTTTTCTGTGTGTTGGTTTTTT } \\
\text { GTGTG }\end{array}$ & 77 \\
\hline
\end{tabular}




\begin{tabular}{|c|c|c|}
\hline terminator & sequence $5^{\prime}>3$ & length \\
\hline Tm.synth.23 & TGTAATGTAATGTAATGTAAAATAAAgtaccetgtgctcaaccagtCATGTGTTTT & 56 \\
\hline Tm.synth.24 & TGTAATGTAATGTAATGTAAAATAAAgtaccetgtgctcaaccagtCATCTGTGTGTTGGTTTTTTGTGTG & 71 \\
\hline Tm.synth. 25 & $\begin{array}{l}\text { TGTAATGTAATGTAATGTAAAATAAAgtaccetgtgctcaaccagtCATGTGTTTTTCTGTGTGTTGGTTTTT } \\
\text { TGTGTG }\end{array}$ & 79 \\
\hline Tm.synth.26 & TGTAATGTAATGTAATGTAAAATAAAtgaattttaagctttCATGTGTTTT & 51 \\
\hline Tm.synth. 27 & TGTAATGTAATGTAATGTAAAATAAAtgaattttaagctttCATCTGTGTGTTGGTTTTTTGTGTG & 66 \\
\hline Tm.synth. 28 & $\begin{array}{l}\text { TGTAATGTAATGTAATGTAAAATAAAtgaattttaagctttCATGTGTTTTTCTGTGTGTTGGTTTTTTGTG } \\
\text { TG }\end{array}$ & 74 \\
\hline Tm.synth.29 & TGTAATGTAATGTAATGTAAAATAAAgtaccetgtgctcaaccagtCACGTGTTATTCATAAGCATT & 67 \\
\hline Tm.synth.30 & TGTAATGTAATGTAATGTAAAATAAAgtaccetgtgctcaaccagtCAGTTGTGTGTGTTG & 61 \\
\hline
\end{tabular}


Figure S1: Representative cytometry plots with HT1080 cells from the same experiment.

WT control:

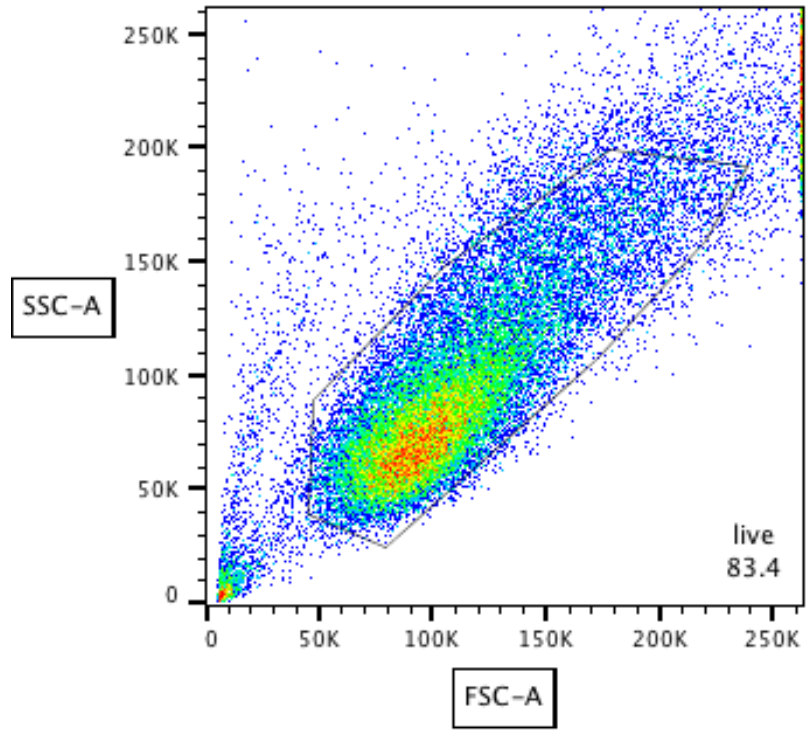

sample: terms_200V_WT_004.fcs gate: Ungated cell count: 34855

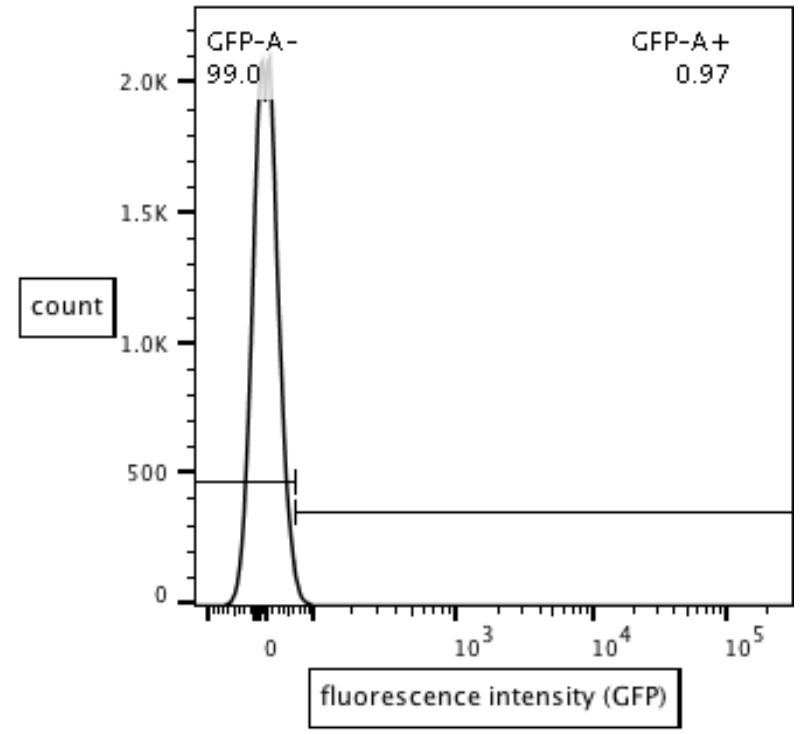

sample: terms_200V_WT_004.fcs gate: live cell count: 29065

No terminator control:

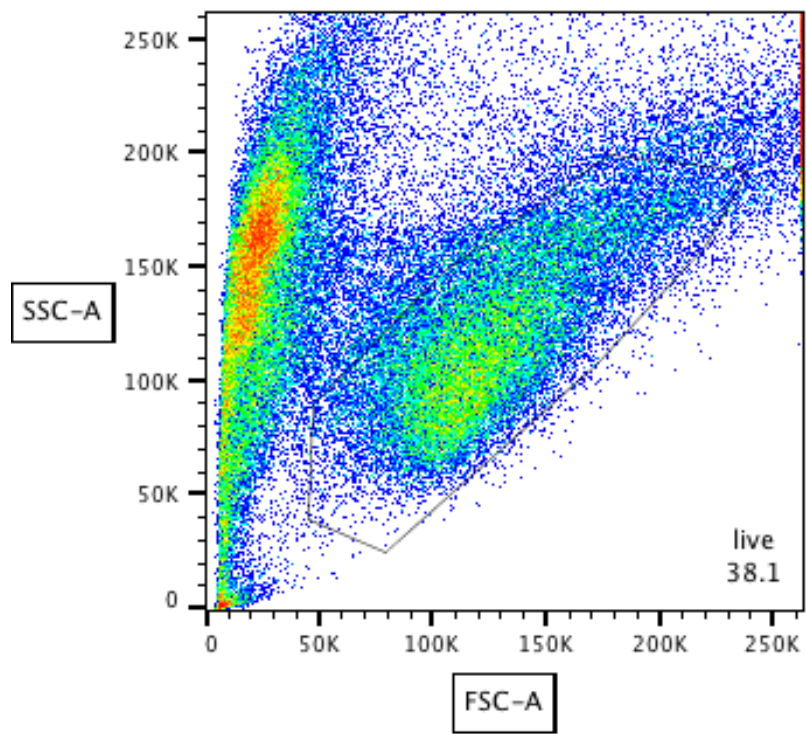

sample: terms_200V_no pA_004.fcs gate: Ungated cell count: 73880

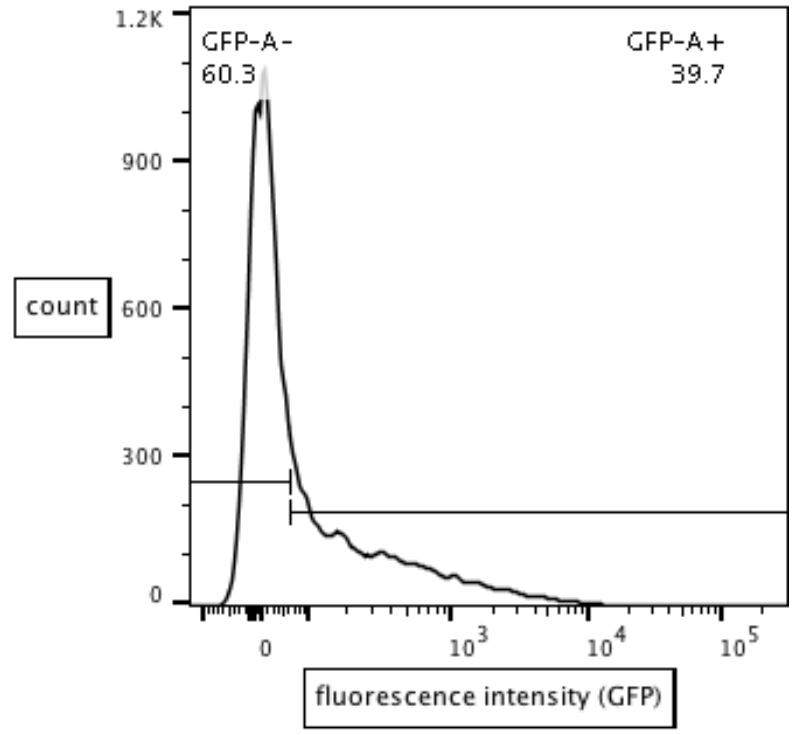

sample: terms_200V_no pA_004.fcs gate: live cell count: 28147 
SV40pA reference control:

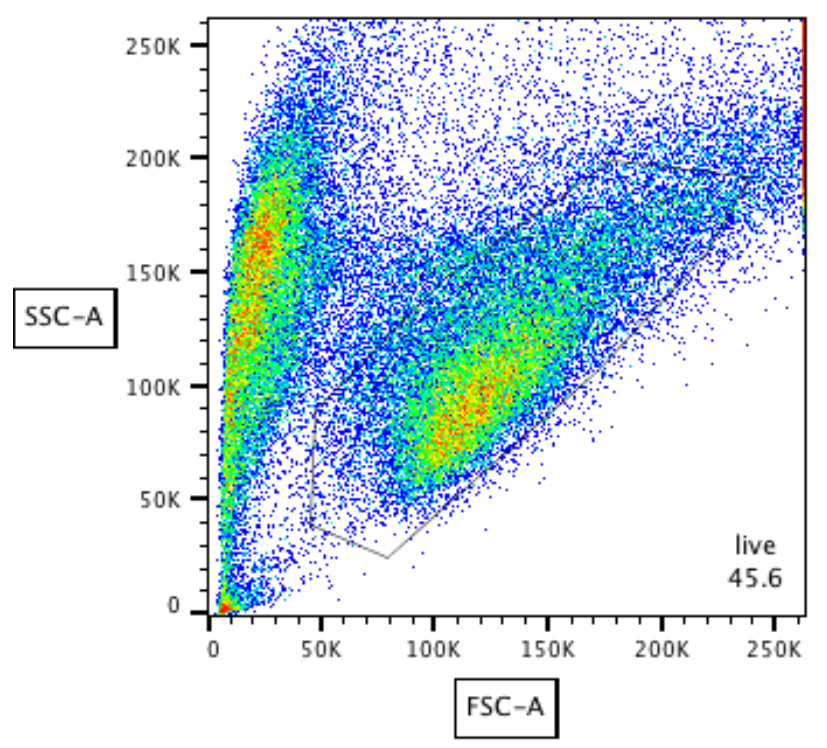

sample: terms_200V_SV40pA_004.fcs gate: Ungated cell count: 61626

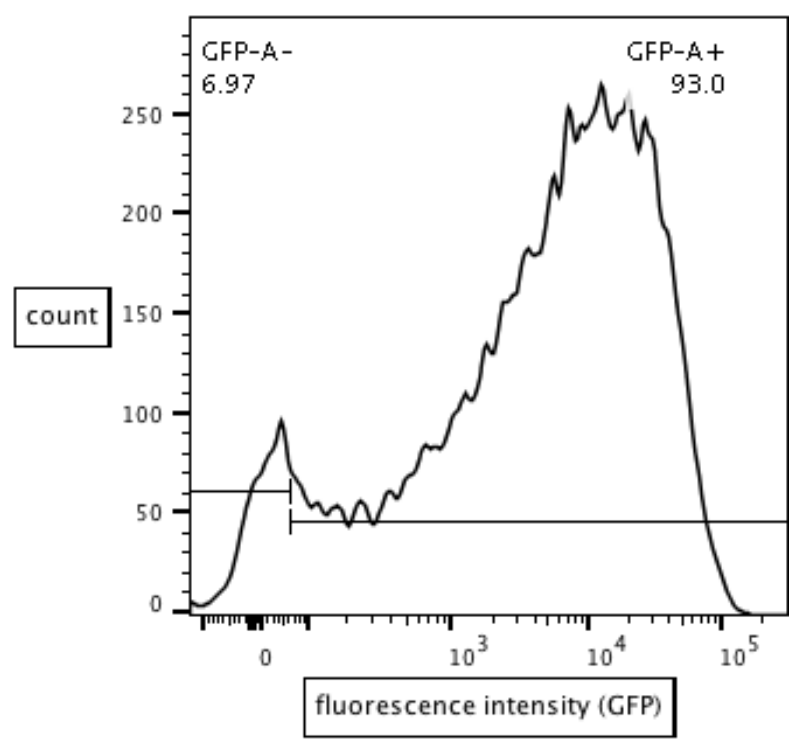

sample: terms_200V_SV40pA_004.fcs gate: live cell count: 28118

T.EEF1A1.1 variant:

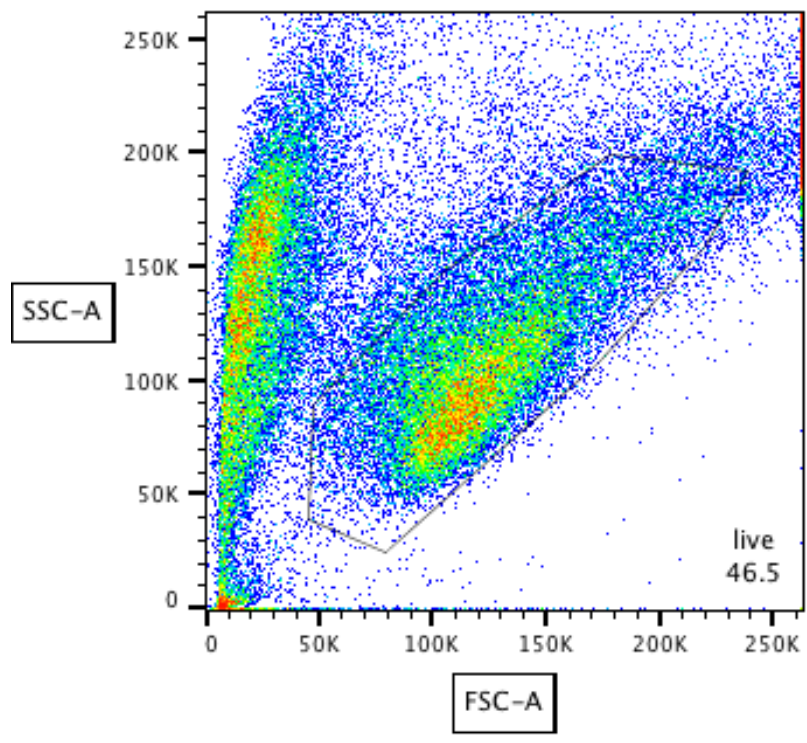

sample: terms_200V_TE-1_002.fcs gate: Ungated cell count: 61006

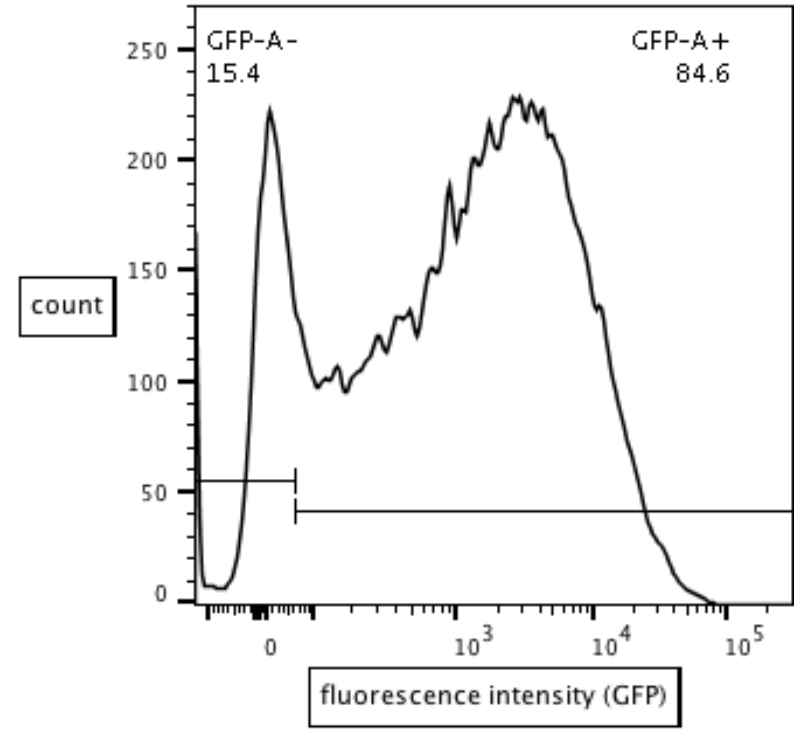

sample: terms_200V_TE-1_002.fcs gate: live cell count: 28368 
T.EEF1A1.2 variant:

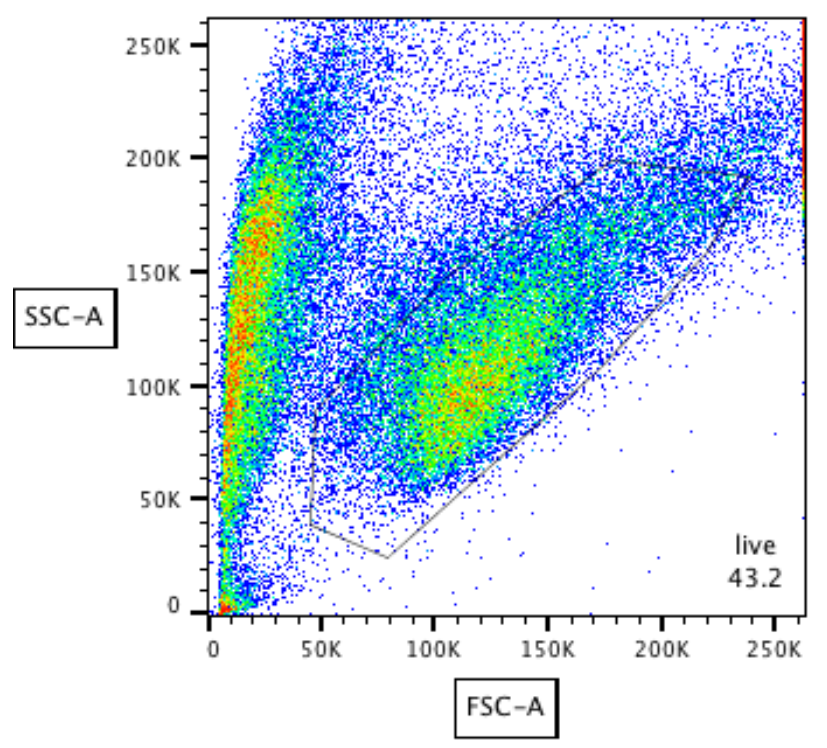

sample: terms_200V_TE-2_002.fcs

gate: Ungated

cell count: 65673

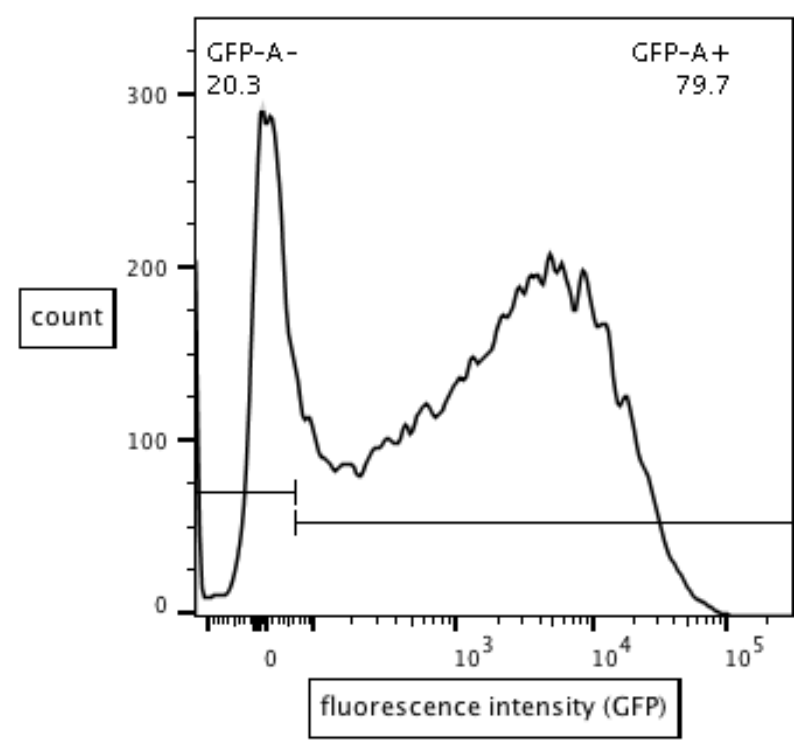

sample: terms_200V_TE-2_002.fcs gate: live cell count: 28338

T.EEF1A1.3 variant:

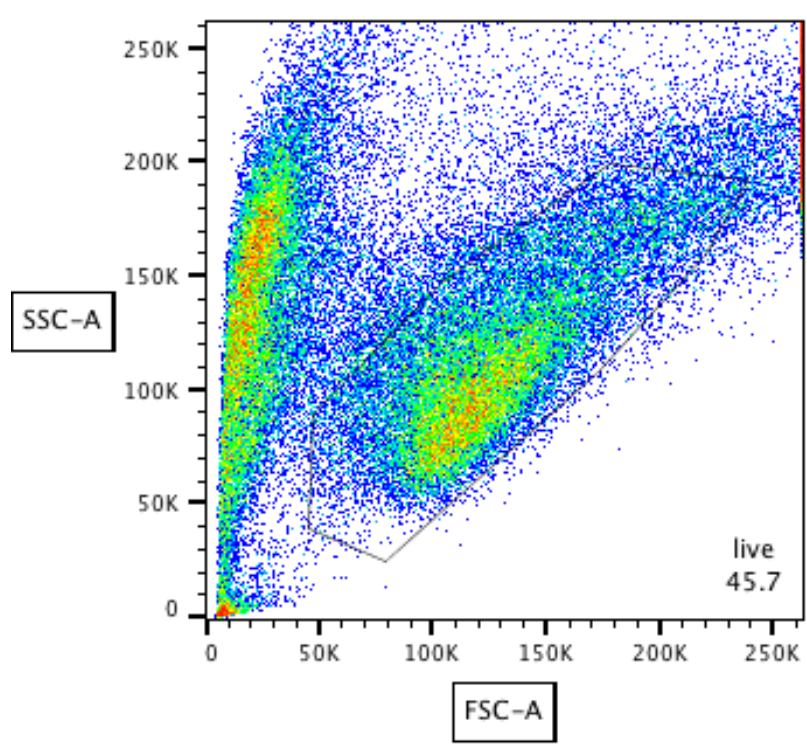

sample: terms_200V_TE-3_002.fcs gate: Ungated cell count: 61482

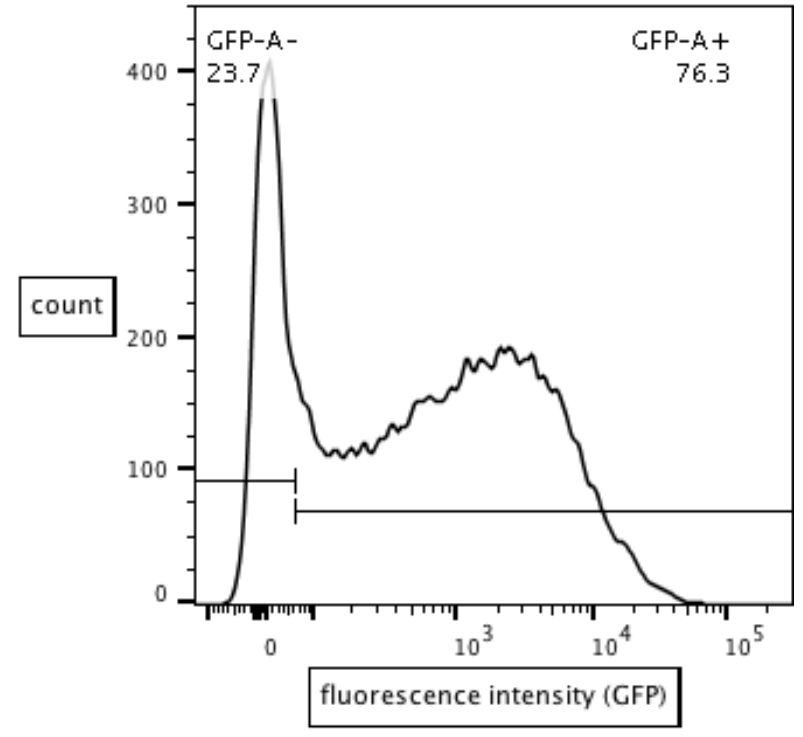

sample: terms_200V_TE-3_002.fcs gate: live cell count: 28112 
T.EEF1A1.4 variant:
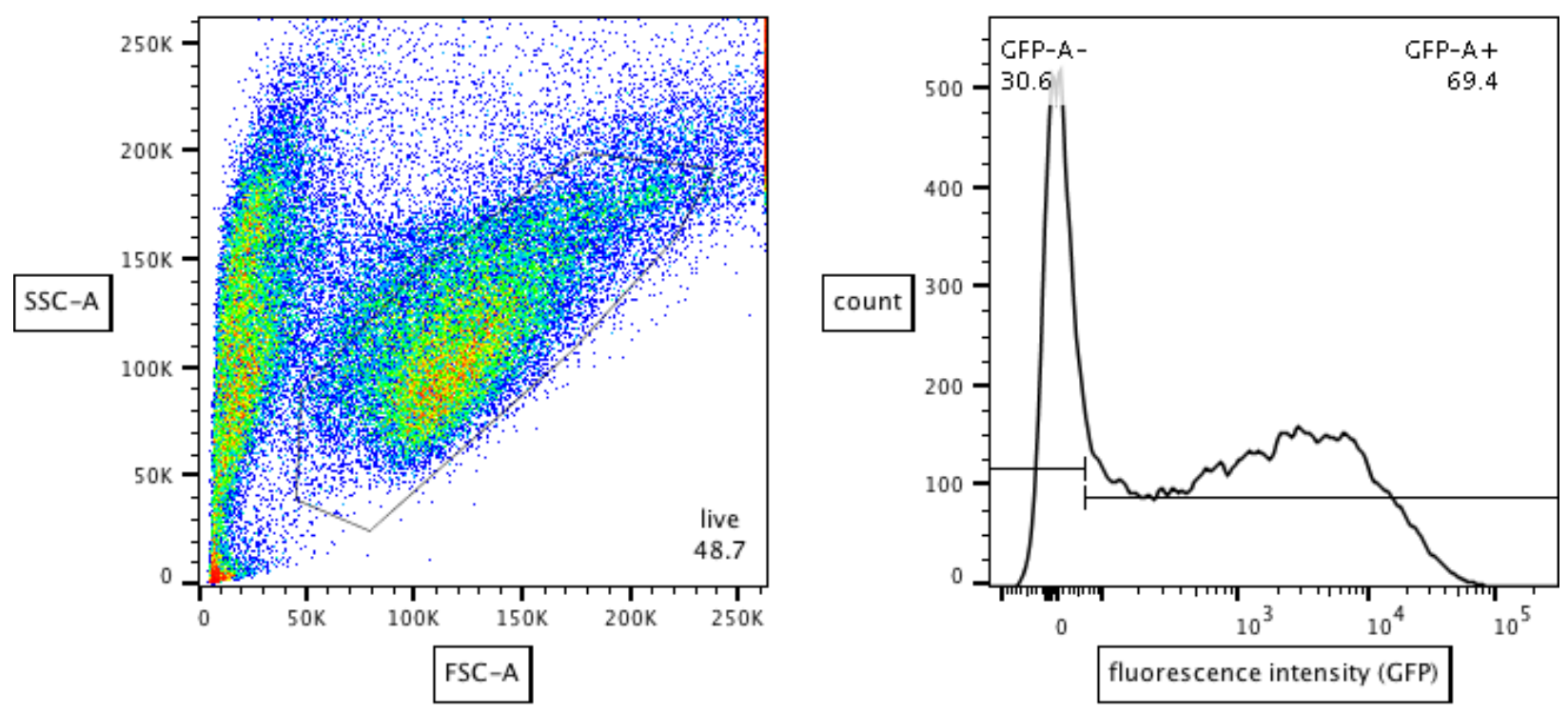

sample: terms_200V_TE-4_002.fcs

gate: Ungated

cell count: 57647

sample: terms_200V_TE-4_002.fcs

gate: live

cell count: 28097 
Figure S2: Representative cytometry plots with HT1080 cells from the same experiment, incorporating a strong constitutive mStrawberry (RFP) expression cassette as a transfection marker on the same plasmid as the hrGFP reporter.

WT control:

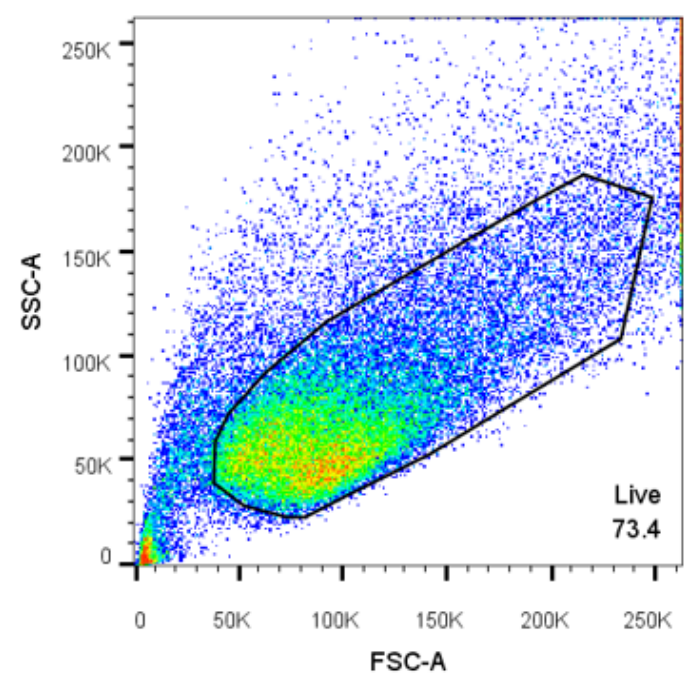

sample: WT control

gate: Ungated

event count: 57600

No terminator control:

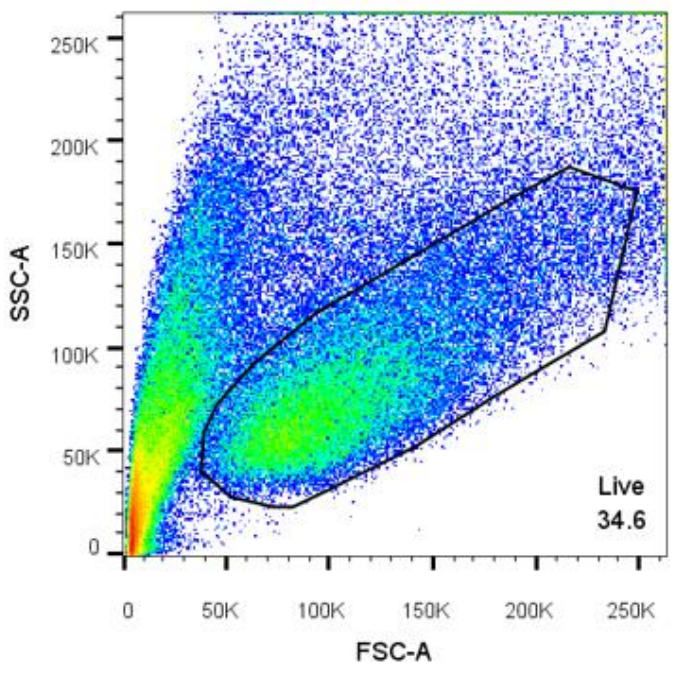

sample: No terminator control gate: Ungated

event count: 124177

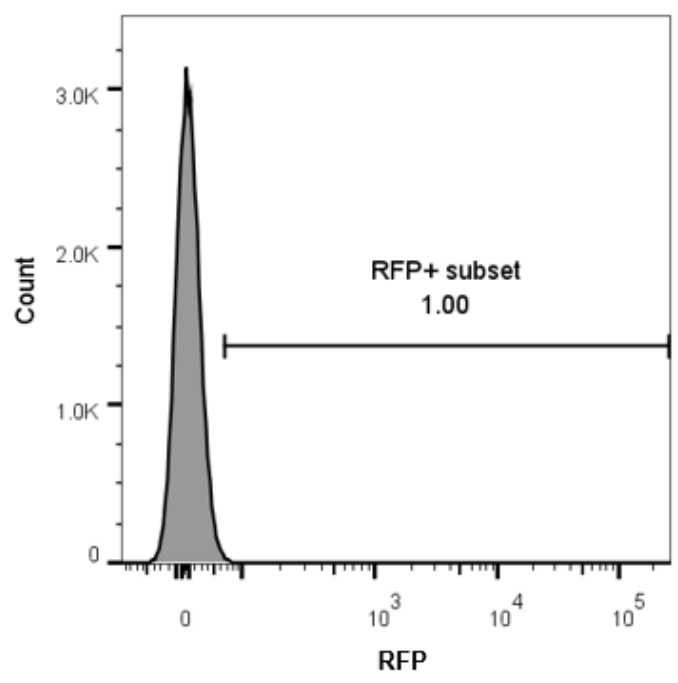

sample: WT control

gate: Live

event count: 42298

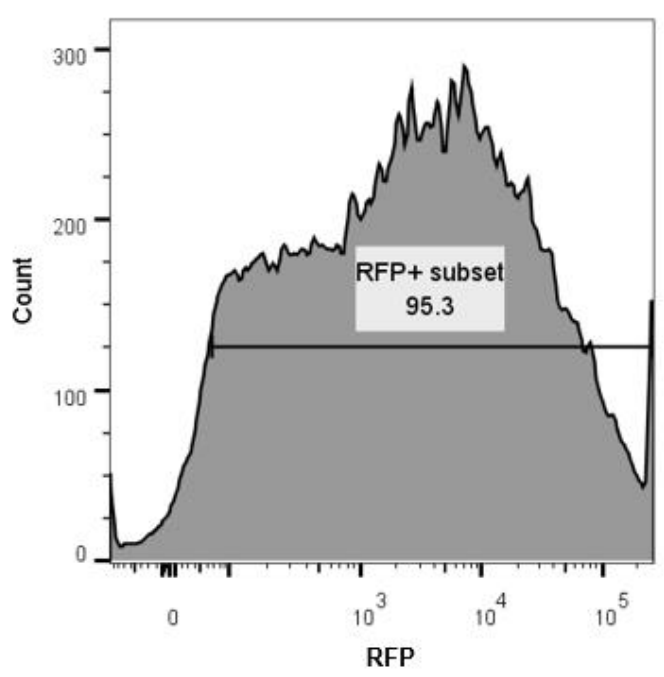

sample: No terminator control gate: Live

event count: 42956 
SV40pA reference control:

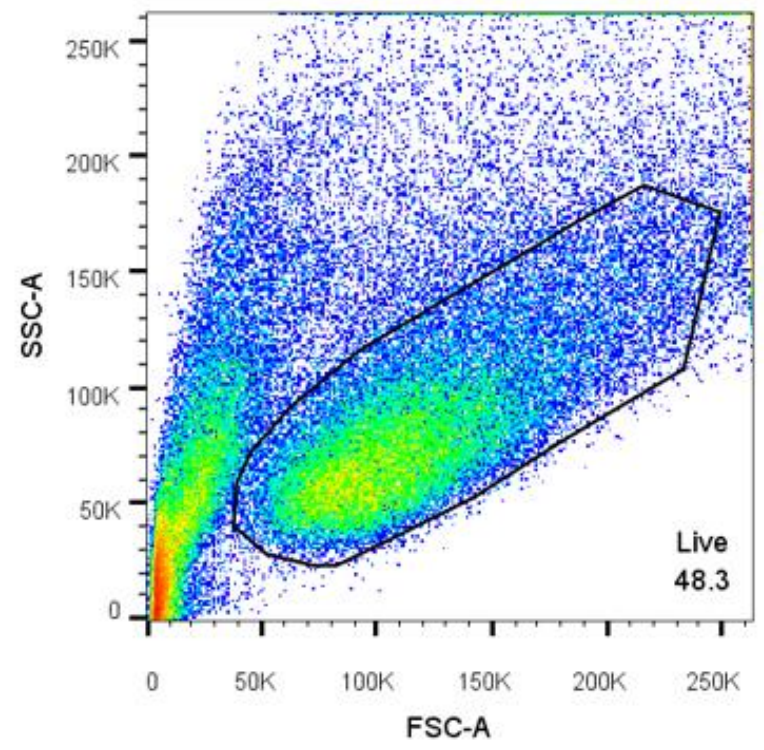

sample: SV40pA reference

gate: Ungated

event count: 86484

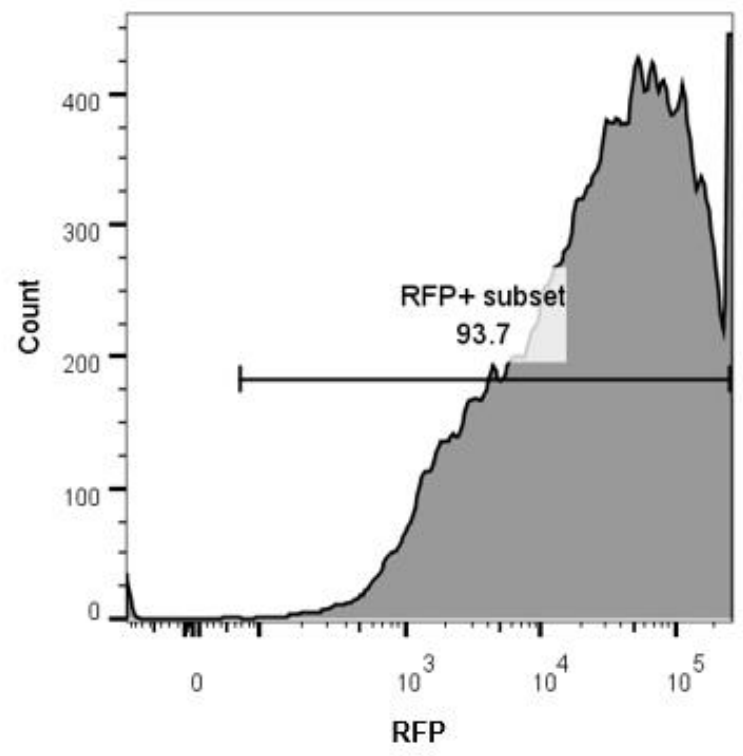

sample: SV40pA reference

gate: Live

event count: 41811

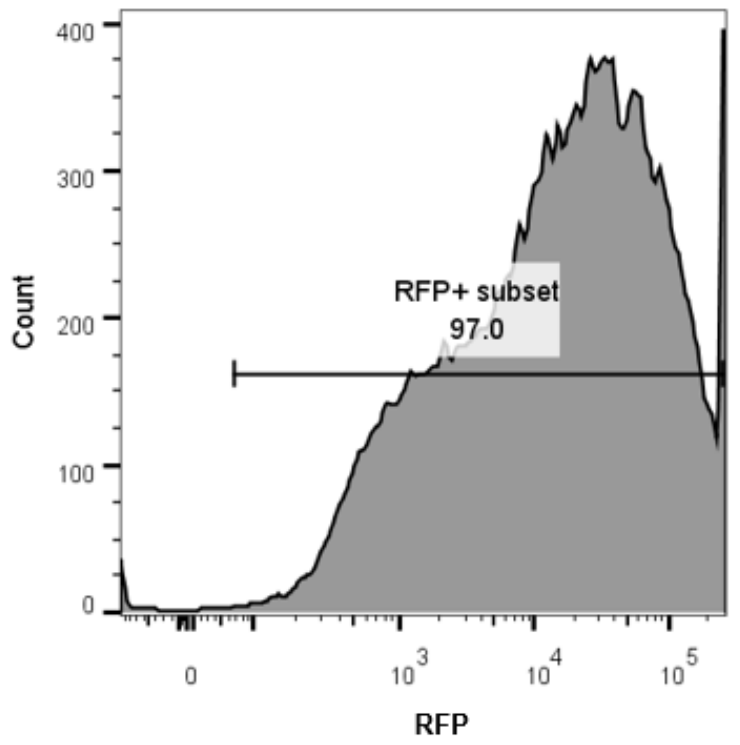

sample: T.GAPDH.1

gate: Live

event count: 41339 
T.GAPDH.2 variant:

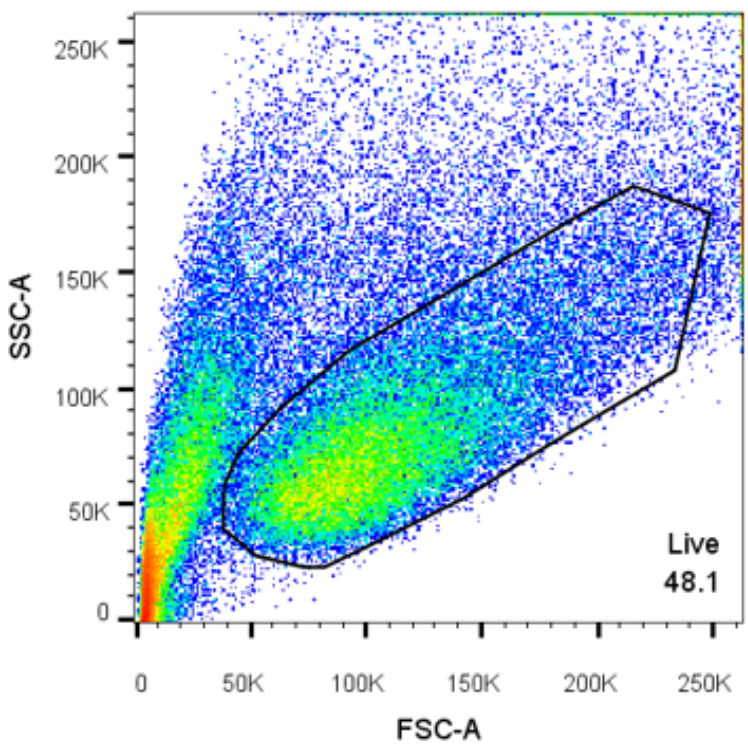

sample: T.GAPDH.2

gate: Ungated

event count: 87542

Tm.synth.02 variant:

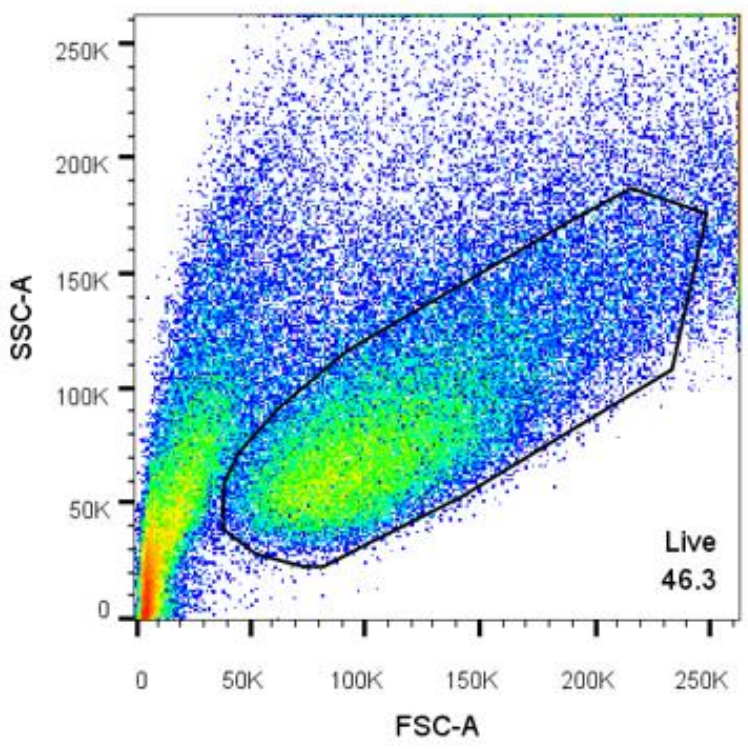

sample : Tm.synth.02

gate: Ungated

event count: 92371

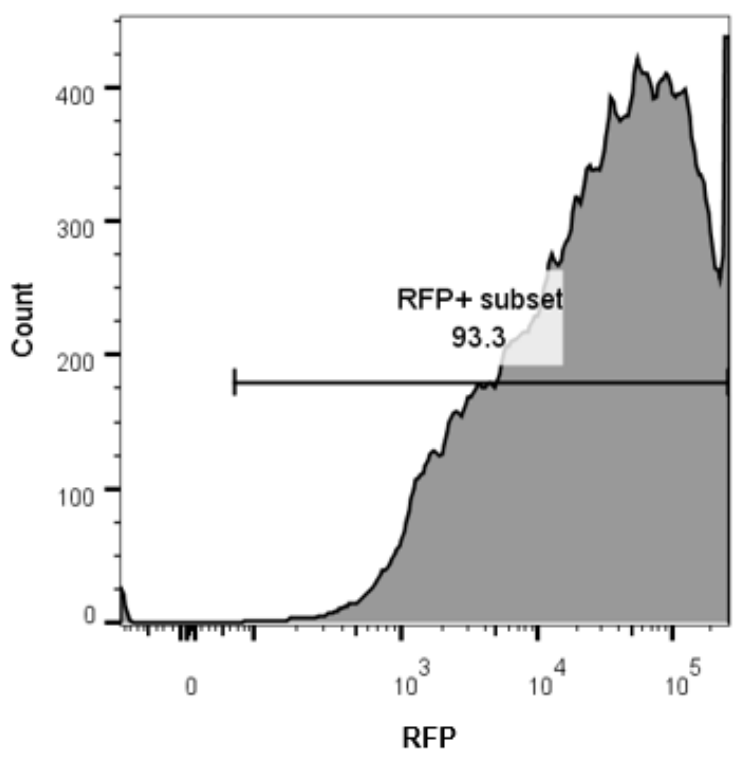

sample: T.GAPDH.2

gate: Live

event count: 42068

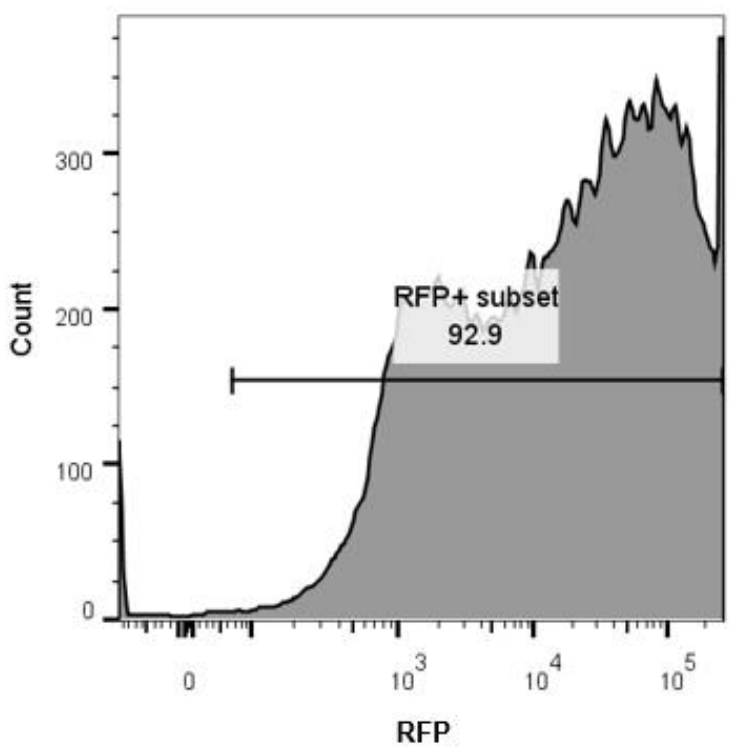

sample: Tm.synth.02

gate: Live

event count: 42809 
Tm.synth.15 variant:

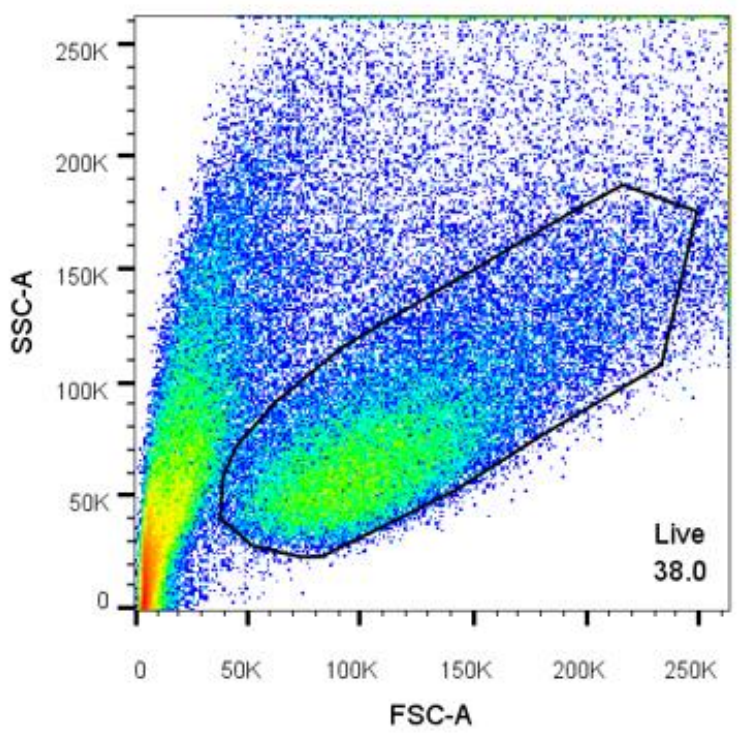

sample: Tm.synth.15

gate: Ungated

event count: 109145

Tm.synth.17 variant:

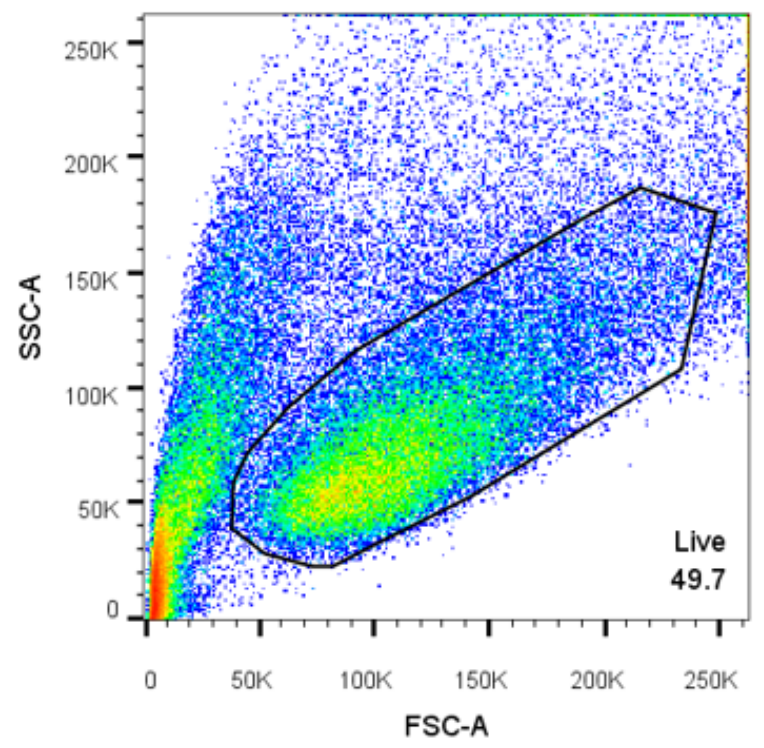

sample: Tm.synth.17 gate: Ungated event count: 83320

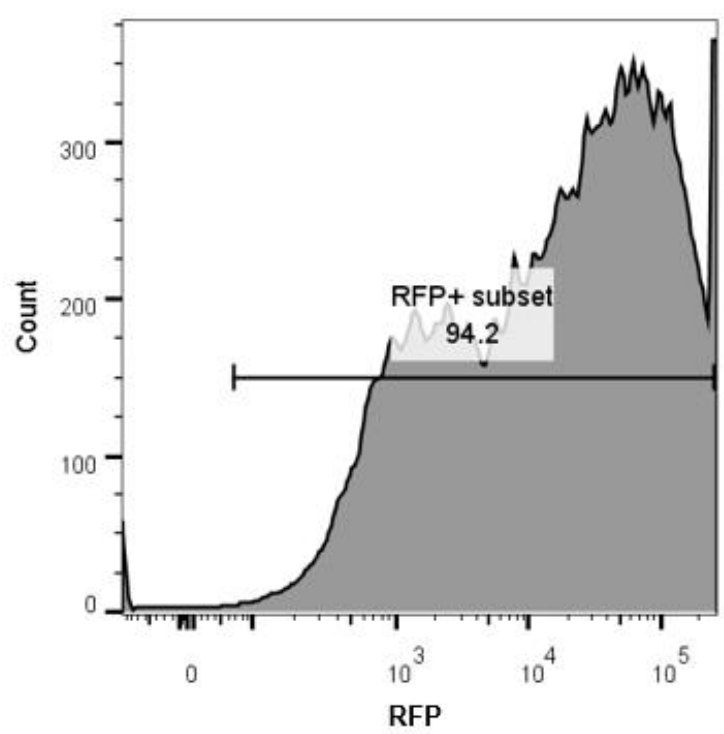

sample: Tm.synth.15

gate: Live

event count: 41489

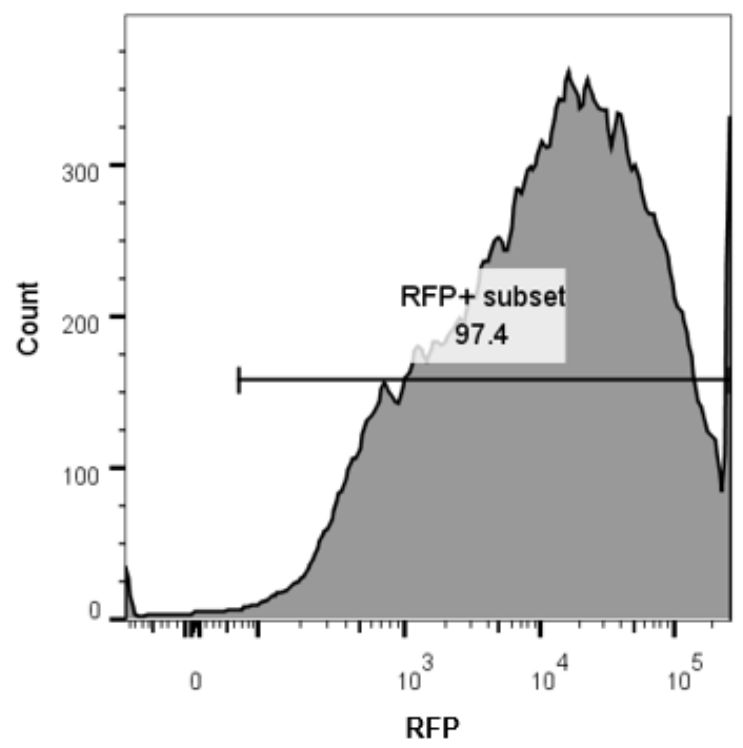

sample: Tm.synth.17

gate: Live

event count: 41430 
Tm.synth. 22 variant:

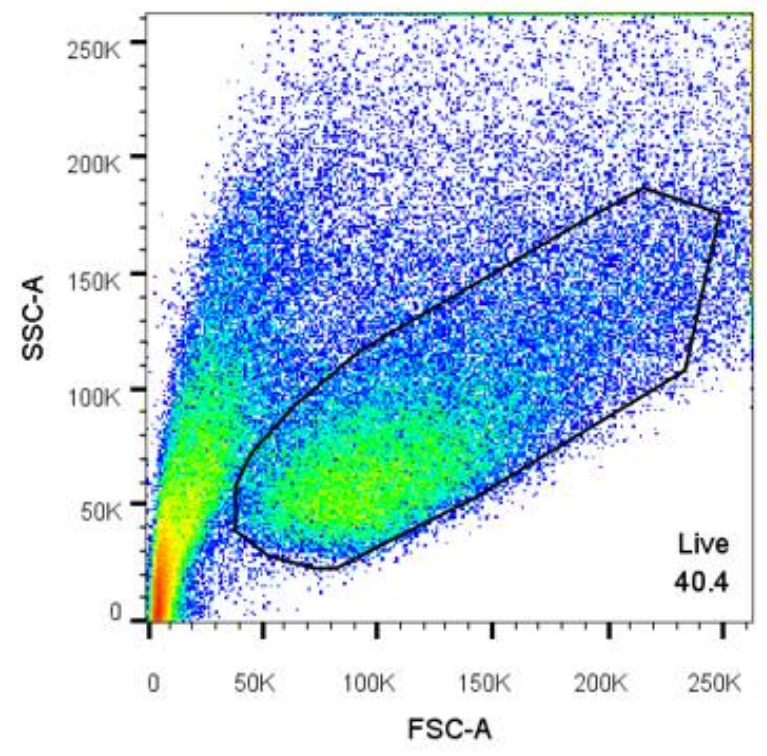

sample: Tm.synth. 22

gate: Ungated

event count: 104142

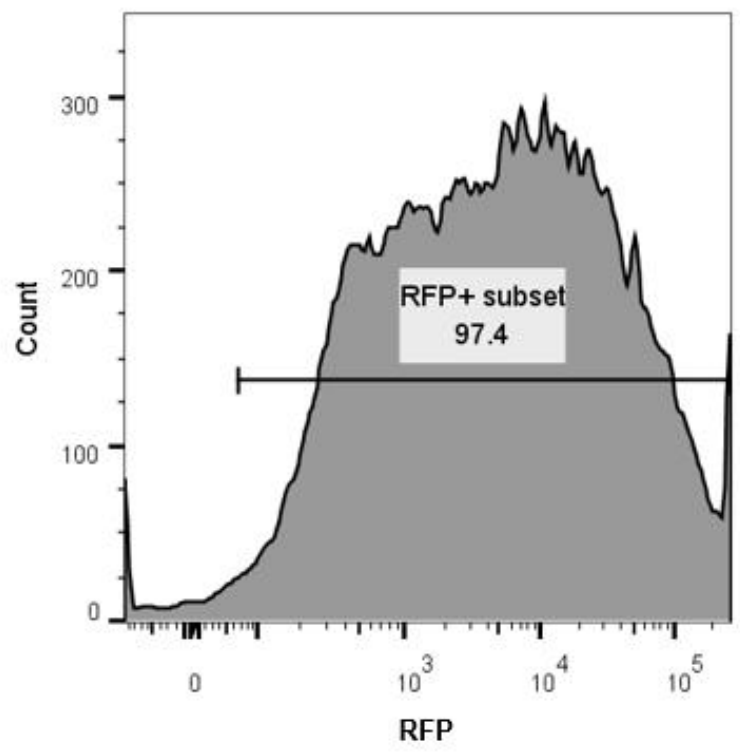

sample: Tm.synth.22

gate:Live

event count: 42088 